\title{
Risk Assessment Chart for Death From Cardiovascular Disease Based on a 19-Year Follow-up Study of a Japanese Representative Population NIPPON DATA80
}

\author{
NIPPON DATA80 Research Group*
}

\begin{abstract}
Background Based on the NIPPON DATA80, risk charts for the probability of death from coronary heart disease (CHD), stroke, and all cardiovascular disease (CVD) were constructed by sex and 10-year age groups. Methods and Results The 9,638 participants were followed-up for 19 years from 1980, excluding 28 individuals without the necessary baseline data and 257 participants with past history of stroke or CHD. Final analysis was performed on 9,353 participants (4,098 men, mean age 50.3 years; 5,255 women, mean age 50.8) using a Cox proportional hazards model. Death probabilities over a 10-year period from CHD, stroke, and all CVD were calculated and displayed as color coding on each chart by combining 10-year age, systolic blood pressure, smoking, and serum total cholesterol and glucose levels. Six different colors corresponding to probabilities of death were displayed on each chart.

Conclusions The original charts based on the findings from NIPPON DATA80 are suitable for assessing CHD, stroke, and all CVD death risk in the general Japanese population. These charts should be used as a health-education tool for lifestyle modification targeting individuals with CVD risk factors. (Circ J 2006; 70: 1249-1255)
\end{abstract}

Key Words: Blood pressure; Cholesterol; Coronary heart disease; Glucose; Smoking; Stroke

A

19-year follow-up study of a Japanese representative population, NIPPON DATA80, revealed that risk factors for stroke, coronary heart disease (CHD), and all cardiovascular disease (CVD) were not different from those of Western societies, ${ }^{1-4}$ although absolute risks for stroke and CHD in Japan were different ${ }^{1-4}$ mainly due to differences in incidence and mortality from stroke and CHD?-10 Japanese individuals had the highest rate of stroke mortality in the world in 1965 but the lowest CHD incidence and mortality among industrialized countries ${ }^{5-10}$

For assessing an individual's risk of stroke, CHD, and all CVD, Framingham CHD risk score, and New Zealand and European charts have been formulated and are now tools used in patient education ${ }^{4,11-13}$ However, a risk assessment chart or score for the Japanese population is necessary because of the differences in CVD morbidity and mortality.

NIPPON DATA80 is a 19-year follow-up study of mortality from stroke, CHD, and all CVD in about 10,000 representative Japanese men and women aged 30 years and older. The purpose of the present study was to construct risk-assessment charts for death from CHD, stroke, and all CVD based on NIPPON DATA80.

\section{Methods}

Population and Follow-up

Complete details of the NIPPON DATA80 study popu-

(Received June 6, 2006; revised manuscript received July 13, 2006; accepted July 21, 2006)

*Members of the research group are listed in the Appendix 1.

Mailing address: Hirotsugu Ueshima, MD, Department of Health Science, Shiga University of Medical Science, Tsukinowa-cho, Seta, Otsu 520-2192, Japan. E-mail: hueshima@belle.shiga-med.ac.jp lation have been described elsewhere ${ }^{1-3,14}$ Subjects of this cohort were participants in the 1980 National Survey on Circulatory Disorders ${ }^{1-3}$ A total of 10,546 communitybased subjects aged 30 years and older in 300 randomly selected health districts throughout Japan participated in the survey. The survey consisted of history-taking, physical examinations, blood tests, and a self-administered questionnaire on lifestyle, including an essential nutrition survey using the food-frequency method. The overall population aged 30 years and older in the 300 participating health districts was 13,771 , and the participation rate in the survey was $76.6 \%(10,546 / 13,771)$, before exclusion for the reasons below. We were able to follow-up 9,638 participants for 19 years, excluding 28 individuals without the necessary baseline data and 257 participants with a past history of stroke or CHD. Thus, we analyzed data from 9,353 participants (4,098 men, mean age 50.3 years; 5,255 women, mean age 50.8) for this study.

To determine cause of death in participants whot died during follow-up, we used the National Vital Statistics. In accordance with Japan's Family Registration Law, all death certificates issued by physicians are forwarded to the Ministry of Health and Welfare via the public health centers in the district of residency. Underlying causes of death were coded according to the $9^{\text {th }}$ International Classification of Disease for the National Vital Statistics until the end of 1994 , and according to the $10^{\text {th }}$ International Classification of Disease from the beginning of $19955^{15}$ We confirmed deaths in each district by computer-matching of data from the Vital Statistics database, using the district, sex, and dates of birth and death as key codes.

Permission to use the National Vital Statistics was obtained from the Management and Coordination Agency, of 
Table 1 Baseline Characteristics of the Study Subjects in 1980, Number of Death From Stroke, Coronary Heart Disease and All Cardiovascular Disease During 19-Year Follow-up, NIPPON DATA80

\begin{tabular}{lcc}
\hline \hline & Men & Women \\
\hline No. of subjects & 4,098 & 5,255 \\
No. of deaths from stroke & 162 & 150 \\
No. of deaths from coronary heart disease & 67 & 65 \\
No. of deaths from all cardiovascular disease & 339 & 339 \\
Age (years) & $50.3 \pm 13.1$ & $50.8 \pm 13.3$ \\
Systolic blood pressure $(\mathrm{mmHg})$ & $138.4 \pm 20.8$ & $133.9 \pm 21.4$ \\
Total cholesterol $(\mathrm{mg} / \mathrm{dl})$ & $186.1 \pm 32.7$ & $190.8 \pm 34.1$ \\
Blood glucose $(\mathrm{mg} / \mathrm{dl})$ & $101.0 \pm 32.4$ & $99.6 \pm 28.7$ \\
\% of those having glucose $\geq 200 \mathrm{mg} / \mathrm{dl}$ & 1.61 & 1.16 \\
Smoker $(\%)$ & 63.3 & 8.8 \\
\hline
\end{tabular}

the national government of Japan. Approval for this study regarding ethics issues was obtained from the Institutional Review Board of the Shiga University of Medical Science (No. 12-18, 2000).

\section{Biochemical and Baseline Examinations}

Baseline examinations were conducted by public health centers. Baseline systolic and diastolic blood pressures (SBP, DBP) were measured by trained operators using a standard mercury sphygmomanometer on the right arm of seated subjects after at least $5 \mathrm{~min}$ of rest.

Subjects were asked to note whether they were current smokers, had quit smoking, or had never smoked. Smokers were asked to note the number of cigarettes smoked each day. Non-fasting blood samples were drawn and centrifuged within $60 \mathrm{~min}$ of collection, and then stored at $-70^{\circ} \mathrm{C}$ until analyses. Total cholesterol was analyzed in a sequential auto-analyzer (SMA12/60; Technicon, Tarrytown, NY, USA) at a single laboratory (Osaka Medical Center for Health Science and Promotion), which is a member of the Cholesterol Reference Method Laboratory Network $(\mathrm{CRMLN}){ }^{16}$ and the precision and accuracy of the measurements of serum cholesterol were certified in the Lipid Standardization Program administered by the Centers for Disease Control and Prevention, Atlanta. Serum concentration of glucose was measured by the cupric-neocuproine method ${ }^{17}$ Original glucose values obtained by the cupricneocuproine method were converted to those of the glucoseoxidase method, which is currently the standard, by use of an equation reported by the same laboratory! 18 Diabetes mellitus (DM) was defined as a serum glucose concentration $\geq 200 \mathrm{mg} / \mathrm{dl}$.

\section{Statistical Analysis}

Complete details of the statistical methods are reported elsewhere. ${ }^{9}$ The outcome event used in the present study was cause-specific death from stroke, CHD, and all CVD. A Cox proportional hazards model was used to determine the probability of death of those with risk factor $x$ at baseline. Survival probability $\mathrm{S}(\mathrm{t}: x)$ at the time t for risk factor $x$ in the Cox regression analysis is given as the following: $\left.\mathrm{S}(\mathrm{t}: x)=\left[\mathrm{S}_{0}(\mathrm{t}: \bar{x})\right] \exp \beta(\bar{x}-x)\right)$, where $\bar{x}$ is population mean of risk factor $x$, and $\mathrm{S}_{0}(\mathrm{t}: \bar{x})$ is survival probability for those with risk factor $\bar{x}$. The 10 -year probability of death for risk factor $x$ was calculated by the following equation: $1-\mathrm{S}$ $(10: x)$

We constructed risk assessment charts for 10-year probability of death due to CHD, stroke, and all CVD in both men and women using traditional risk factors (ie, SBP, smoking habit, serum total cholesterol and serum glucose). SBP was classified into 5 categories: (1) 100$119 \mathrm{mmHg}$, (2) $120-139 \mathrm{mmHg}$, (3) $140-159 \mathrm{mmHg}$, (4) $160-179 \mathrm{mmHg}$ and (5) $180-199 \mathrm{mmHg}$. Smoking was divided into 2 categories: non-current smoker and current smoker. Serum total cholesterol was classified into 6 categories: (1) $160-179 \mathrm{mg} / \mathrm{dl}$, (2) $180-199 \mathrm{mg} / \mathrm{dl}$, (3) $200-$ $219 \mathrm{mg} / \mathrm{dl}$, (4) $220-239 \mathrm{mg} / \mathrm{dl}$, (5) $240-259 \mathrm{mg} / \mathrm{dl}$, and (6) 260-279 mg/dl. Casual serum glucose level was divided into 2 categories: $<200 \mathrm{mg} / \mathrm{dl}$ and $\geq 200 \mathrm{mg} / \mathrm{dl}$. These cutoff points were based on either practical considerations or guideline recommendations from the Hypertension Treatment Guidelines 2004 in Japan and the Treatment of Atherosclerosis in Japan?20,21 Ten-year death probabilities from stroke, CHD, and all CVD were calculated and displayed as color coding on each chart, combining 10-year age, systolic blood pressure, smoking, and serum total cholesterol and glucose levels. Six different colors were displayed on each chart corresponding to the following probabilities of death: $<0.5 \%, 0.5-1 \%, 1-2 \%, 2-5 \%, 5-10 \%$ and $\geq 10 \%$ for CHD and stroke, and $<1.0 \%, 1-3 \%, 3-7 \%, 7-15 \%, 15-$ $30 \%$ and $\geq 30 \%$ for all CVD. SAS version 8.02 for Windows (SAS Institute, Cary, NC) was used for estimating a regression coefficient of, and the survival probability $\mathrm{S}_{0}(\mathrm{t}: \bar{x})$ for those with the population mean $\bar{x}$ of a risk factor.

\section{Results}

During the 19-year follow-up from 1980, the number of deaths from stroke and CHD was 312 and 132, respectively (Table 1), and death rates were 1.93 and 0.81 per 1,000 person-years. Average age for men and women at baseline was 50.3 and 50.8 years, respectively. Although SBP was higher in men than in women, serum total cholesterol was higher in women than in men $(190.8 \mathrm{mg} / \mathrm{dl} \mathrm{vs} 186.1 \mathrm{mg} / \mathrm{dl})$. Prevalence of casual glucose level $\geq 200 \mathrm{mg} / \mathrm{dl}$ was slightly higher in men than in women (1.61\% vs $1.16 \%)$. Smoking rate in men was far greater than in women, reflecting the relatively high smoking rate of Japanese men in 1980.

Table 2 Age-Specific Mortality per 1,000 Person-Years, 19-Year Follow-up, NIPPON DATA80

\begin{tabular}{|c|c|c|c|c|c|c|}
\hline \multirow{2}{*}{$\begin{array}{c}\text { Age } \\
\text { (years) }\end{array}$} & \multicolumn{2}{|c|}{ Stroke } & \multicolumn{2}{|c|}{$C H D$} & \multicolumn{2}{|c|}{$A l l C V D$} \\
\hline & Men & Women & Men & Women & Men & Women \\
\hline $30-39$ & 0.15 & 0.12 & 0.05 & 0.00 & 0.35 & 0.20 \\
\hline $40-49$ & 0.35 & 0.32 & 0.50 & 0.12 & 1.09 & 0.68 \\
\hline $50-59$ & 1.73 & 0.76 & 0.80 & 0.22 & 3.83 & 1.69 \\
\hline $60-69$ & 5.57 & 2.77 & 2.07 & 1.89 & 10.81 & 6.97 \\
\hline$\geq 70$ & 18.43 & 14.30 & 6.06 & 5.28 & 37.61 & 31.33 \\
\hline
\end{tabular}

CHD, coronary heart disease; $C V D$, cardiovascular disease. 

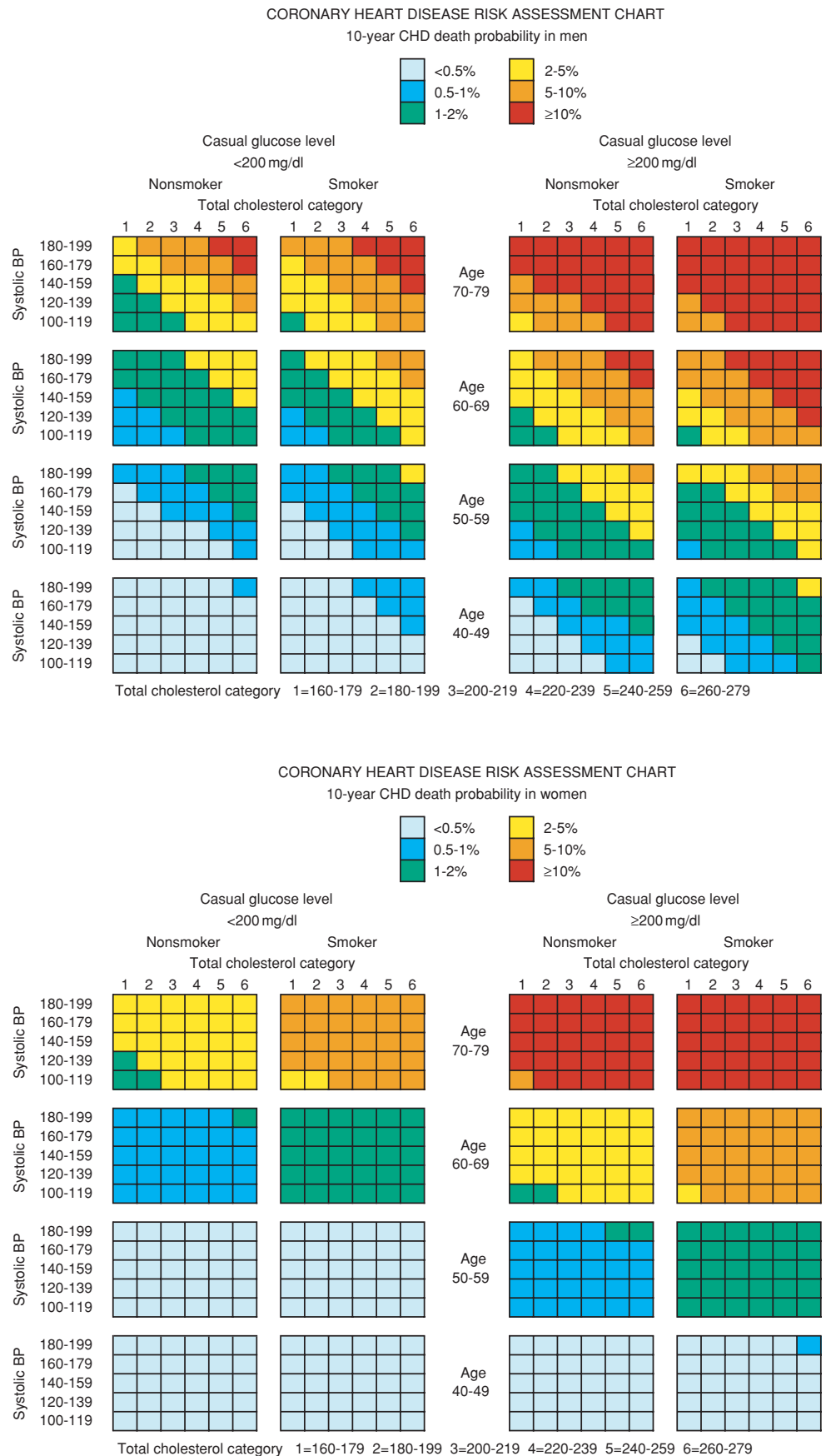

Fig 1. Risk assessment chart for 10-year probability of death due to coronary heart disease in men, NIPPON DATA80. Ten-year probability of death was calculated based on individual risk assessment using sex, age, systolic blood pressure (BP), serum total cholesterol, serum glucose and smoking habit. Someone with any of the cardiovascular disease risk factors, despite belonging to the lowest risk group (light blue) should undergo risk factor modification by non-pharmacological and/or pharmacological treatment. CHD, coronary heart disease.
Fig 2. Risk assessment chart for 10-year probability of death due to coronary heart disease in women, NIPPON DATA80. Ten-year probability of death was calculated based on individual risk assessment using sex, age, systolic blood pressure (BP), serum total cholesterol, serum glucose and smoking habit. Someone with any of the cardiovascular disease risk factors, despite belonging to the lowest risk group (light blue) should undergo risk factor modification by non-pharmacological and/or pharmacological treatment. CHD, coronary heart disease.
Death rates for stroke and CHD by age group at entry in 1980 are shown in Table 2. Stroke death rate was around 3 -fold higher than the CHD death rate among subjects aged 70 years and older. Stroke mortality for men increased with age; however, CHD mortality rate after the age of 70 years was similar for both sexes, although the mortality rate was much higher in men than in women under the age of 70 .

A Cox proportional hazards model was used to determine 10-year probability of death due to CHD, stroke, and all CVD, taking into account baseline risk factors such as age, SBP, serum total cholesterol, smoking, and serum glucose. By using the coefficients from the Cox model and the 10year death probability for mean levels of risk factors (age, smoking, total cholesterol and casual glucose), risk assessment charts were constructed for both sexes for the proba- bility of death within 10 years from CHD, stroke, or all CVD. The 10-year probability of death was determined for various combinations of baseline risk-factor levels. Figs 16 show the results, with a 6-color gradient from the highest (red) to the lowest (light blue) probability of death within 10 years.

In Fig 1 for male CHD risk assessment, if a man aged 65 years had a SBP of $164 \mathrm{mmHg}$, a smoking habit, a glucose level of $210 \mathrm{mg} / \mathrm{dl}$, and a serum total cholesterol of $240 \mathrm{mg} / \mathrm{dl}$, his CHD death probability within 10 years would be $\geq 10 \%$, as shown in red. If this subject quit smoking without any other risk factor changes, his CHD death probability would be $<10 \%$, as shown by the yellowbrown color.

The chart for 10-year stroke death probability was some- 

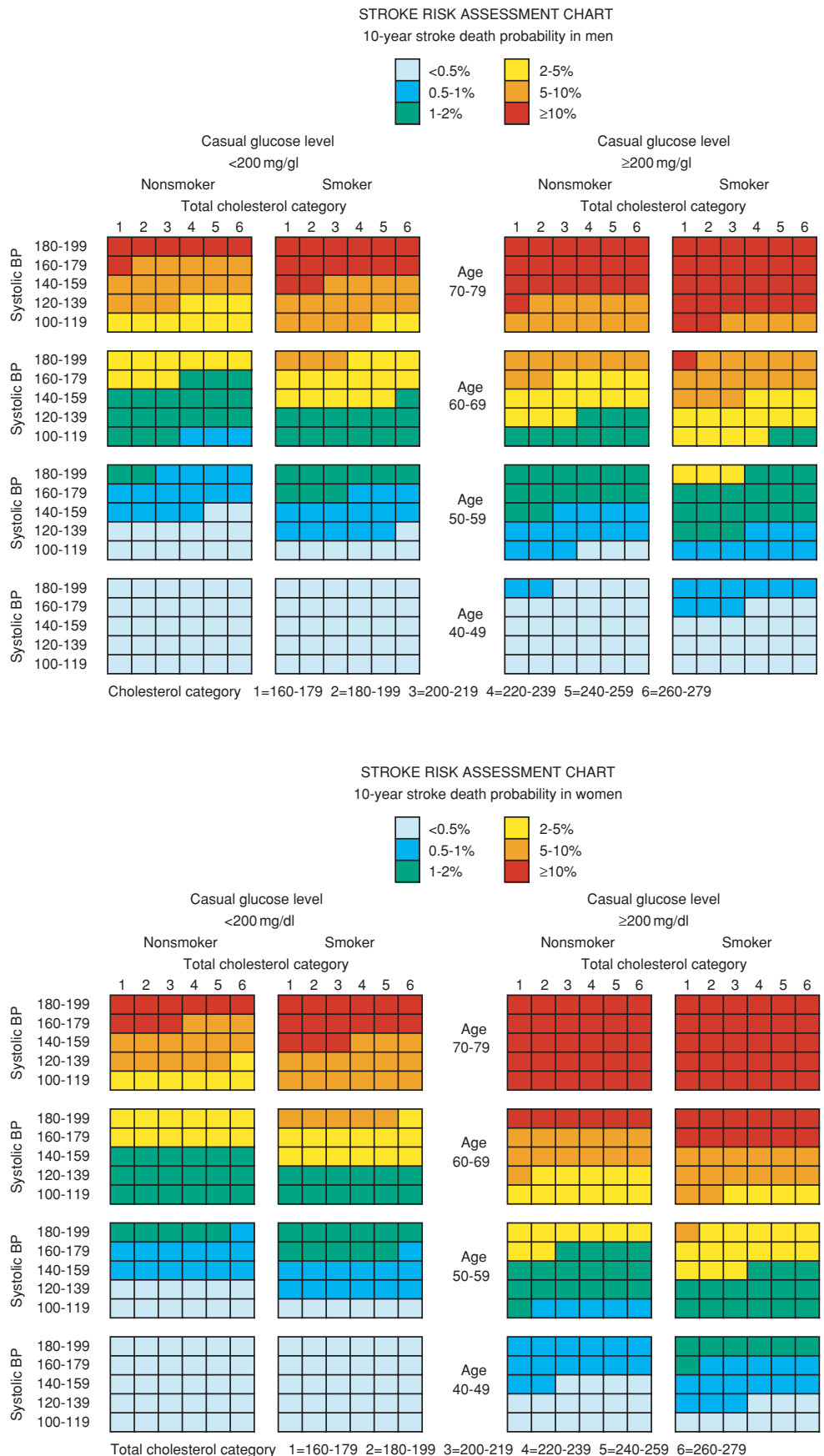

Fig 3. Risk assessment chart for 10-year probability of death due to stroke in men, NIPPON DATA80. Tenyear probability of death was calculated based on individual risk assessment using sex, age, systolic blood pressure (BP), serum total cholesterol, serum glucose and smoking habit. Someone with any of the cardiovascular disease risk factors, despite belonging to the lowest risk group (light blue) should undergo risk factor modification by non-pharmacological and/or pharmacological treatment.
Fig 4. Risk assessment chart for 10-year probability of death due to stroke in women, NIPPON DATA80. Tenyear probability of death was calculated based on individual risk assessment using sex, age, systolic blood pressure (BP), serum total cholesterol, serum glucose and smoking habit. Someone with any of the cardiovascular disease risk factors, despite belonging to the lowest risk group (light blue) should undergo risk factor modification by non-pharmacological and/or pharmacological treatment. what different from the CHD death probability chart for both men and women. For CHD death the color (probability) gradient continued from the lower-left to the upperright, whereas the color gradient for stroke death was not as remarkable as it was for CHD. For all CVD, the chart was a cross between the charts for death risk due to CHD and to stroke. Again, the color gradient tended to proceed from the lower-left to the upper-right, although this gradient was weaker in all CVD than in CHD.

\section{Discussion}

Based on a 19-year follow-up study in a Japanese representative population, we constructed 10 -year death probability charts for CHD, stroke, and all CVD, with a 6-color gradient showing 10-year probability of death. A major strength of these charts is that the risk of death was estimated using traditional risk factors based on a cohort study of a representative Japanese population. Our original charts created in this study may be more suitable in terms of generalization for Japanese people than others based on the modified Framingham CHD risk core, or a local cohort study, 11-13,22 although these charts also contained a highdensity lipoprotein cholesterol variable.

The risk assessment charts presented here show 10-year death probabilities, but not incidence probability. Therefore, the absolute death rate in these charts is lower than that of incidence. However, using a 6-color gradient from the highest (red) to the lowest (light blue) probability of death allows individuals to see their own position on the chart 


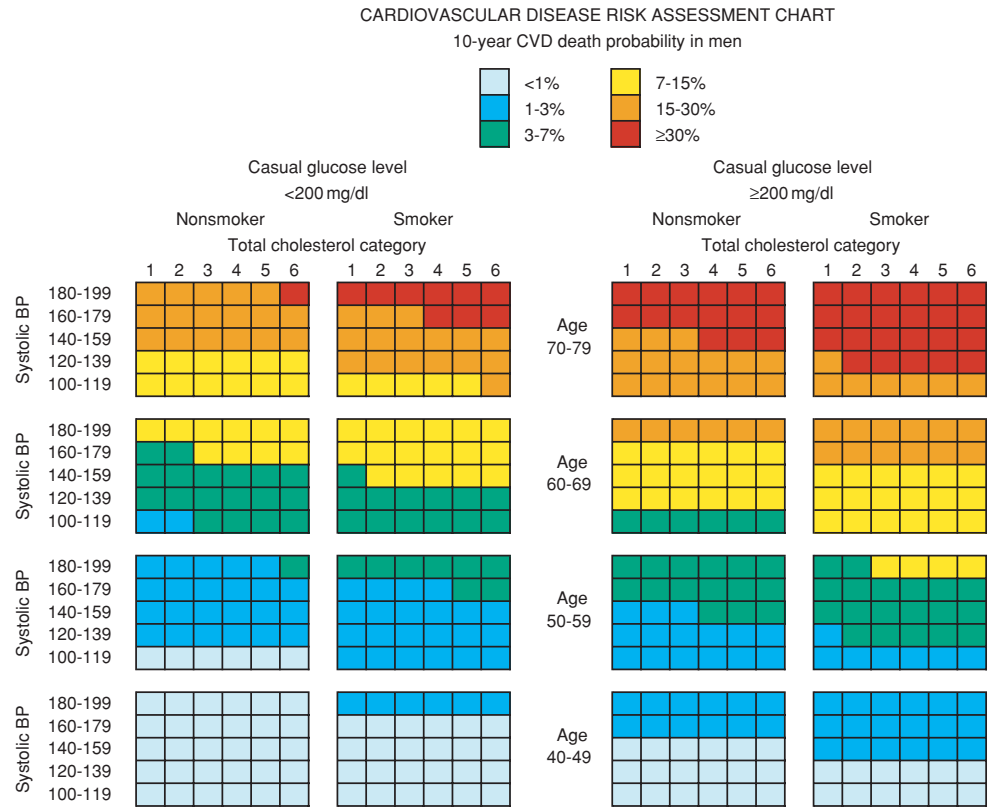

Total cholesterol category $1=160-179 \quad 2=180-199 \quad 3=200-219 \quad 4=220-239 \quad 5=240-259 \quad 6=260-279$

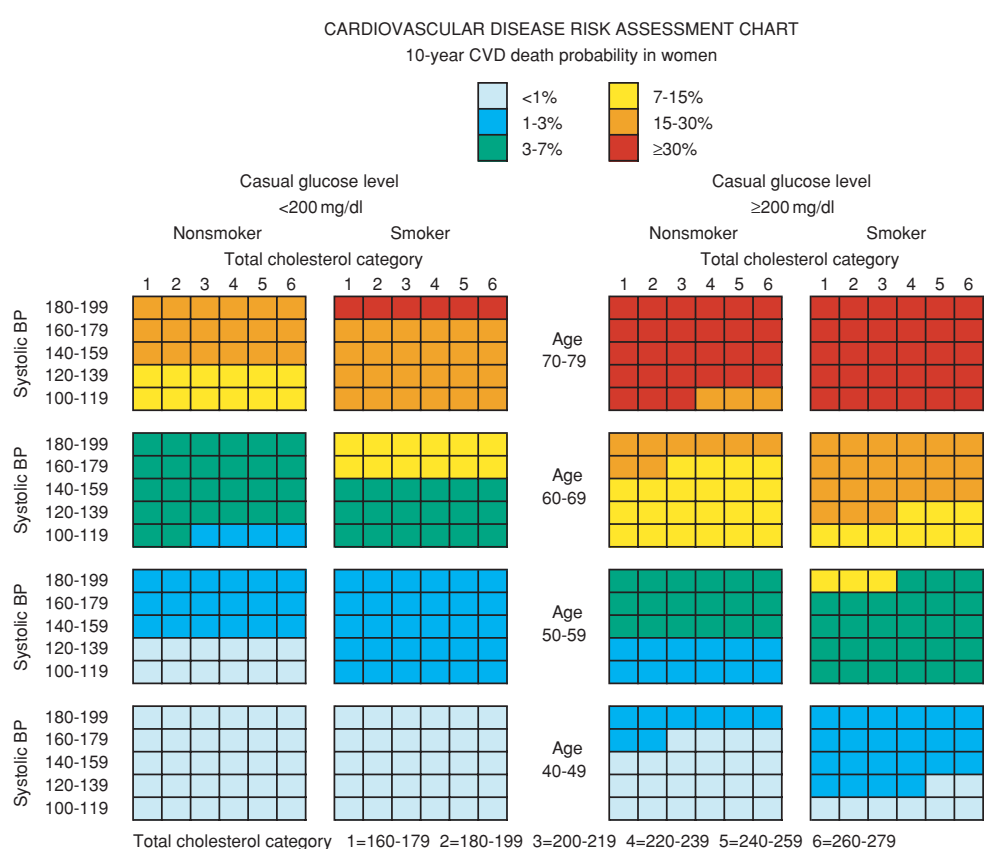

Fig 5. Risk assessment chart for 10-year probability of death due to all cardiovascular disease in men, NIPPON DATA80. Ten-year probability of death was calculated based on individual risk assessment using sex, age, systolic blood pressure (BP), serum total cholesterol, serum glucose and smoking habit. Someone with any of the cardiovascular disease (CVD) risk factors, despite belonging to the lowest risk group (light blue) should undergo risk factor modification by nonpharmacological and/or pharmacological treatment.
Fig 6. Risk assessment chart for 10-year probability of death due to all cardiovascular disease in women, NIPPON DATA80. Ten-year probability of death was calculated based on individual risk assessment using sex, age, systolic blood pressure (BP), serum total cholesterol, serum glucose and smoking habit. Someone with any of the cardiovascular disease (CVD) risk factors, despite belonging to the lowest risk group (light blue) should undergo risk factor modification by nonpharmacological and/or pharmacological treatment. and easily understand their own risk level.

We estimated risk using an age range of 40-79 years and found that men and women in their $40 \mathrm{~s}$ and $50 \mathrm{~s}$ had a lower probability of death even if they had traditional risk factors (ie, smoking and hypertension). In contrast, men and women in their $70 \mathrm{~s}$ had the highest probability of death (yellow, orange and red colors). If we had performed separate risk analysis on each 10-year age range, we could have identified the risk within any given 10-year age range. However, in this study, we followed a similar method of risk assessment as the Framingham CHD risk assessment score 4 and others. ${ }^{12}$ It should be understood that the charts presented here do not alleviate the need to control risk factors in younger individuals, despite their lower probability of death. For example, hypertension should be controlled in everyone by lifestyle modification, and by drug treatment, if necessary. If we ignore hypertension in younger individuals because of their lower risk of mortality from CHD and stroke, such individuals will have increased risk of mortality as they age. This principle also applies to modification of all traditional risk factors, such as high serum cholesterol, smoking, and DM.

Many epidemiologic studies in Japan, including NIPPON DATA80 and 90, have revealed risk factors for stroke, CHD, all heart disease, and all CVD!-3,14,18,20-32 These risks for a specific disease are shown as either relative or absolute. Although absolute and relative risks are useful for health education, the risk assessment charts developed in this study should provide an improved health-education tool. A health professional can give more effective health 
education to individuals using the charts rather than simply informing them of their relative or absolute risk of dying from a particular disease.

The CHD risk assessment chart resulting from this study was clearly graded but the stroke chart was not, mainly because serum total cholesterol was not a risk factor for stroke death, which has has been well documented in several cohort studies?,26,29,33-35 As an epidemiological finding, stroke incidence and mortality in the general Japanese population varied directly with blood pressure but inversely with total serum cholesterol levels.,6-8,10,14,20,21,24,28,29,33-35

In conclusion, our risk assessment charts by sex for CHD, stroke, and all CVD can be used as a health-education tool to show risk of death in Japanese individuals. We hope that these charts are used not only in the clinic but also in the community and industrial health sectors.

\section{Acknowledgments}

This study was supported by a Grant-in-Aid from the Ministry of Health and Welfare under the auspices of Japanese Association for Cerebrocardiovascular Disease Control, a Research Grant for Cardiovascular Diseases (7A-2) from the Ministry of Health, Labour and Welfare, and a Health and Labour Sciences Research Grant, Japan (Comprehensive Research on Aging and Health: H11-Chouju-046, H14-Chouju-003, H17Сhouju-012).

\section{References}

1. NIPPON DATA80 Research Group. Impact of elevated blood pressure on mortality from all causes, cardiovascular diseases, heart disease and stroke among Japanese: 14 year follow-up of randomly selected population from Japanese (Nippon Data80). J Hum Hyperten 2003; 17: 851-857.

2. Okamua T, Kadowaki T, Hyakawa T, Kita Y, Okayama A, Ueshima H, NIPPON DATA80 Research Group. What cause of mortality can we predict by cholesterol screening in the Japanese general population? J Intern Med 2003; 253: 169-180.

3. Ueshima H, Choudhury SR, Okayama A, Hayakawa T, Kita Y, Kadowaki T, et al; NIPPON DATA80 Research Group. Cigarette smoking as a risk factor for stroke death in Japan. Stroke 2004; 35: $1836-1841$

4. Wilson PWF, D'Agostino RB, Levy D, Belanger AM, Silbershatz H, Kannel WB. Prediction of coronary heart disease using risk factor categories. Circulation 1998; 97: 1873-1847.

5. Uemura K, Pisa Z. Trends in cardiovascular disease mortality in industrialized countries since 1950. World Health Stat Q 1988; 41: $155-178$.

6. Ueshima H, Tatara K, Asakura S. Declining mortality from ischemic heart disease and changes in coronary risk factors in Japan, 19561980. Am J Epidemiol 1987; 125: 62-72.

7. Kita Y, Okayama A, Ueshima H, Wada M, Choudhury SR, Bonita R, et al. Stroke incidence and case fatality in Shiga, Japan 1989-1993. Int J Epidemiol 1999; 28: 1059-1065.

8. Yoshida M, Kita Y, Nakamura Y, Nozaki A, Okayama A, Sugiura H, et al. Incidence of acute myocardial infarction in Takashima, Shiga, Japan. Circ J 2005; 69: 404-408.

9. Ueshima H. Trends in Asia. In: Marmot M, Elliott P, editors. Coronary heart disease epidemiology: From aetiology to public health. Oxford; 2005; $102-112$.

10. Ueshima H. Changes in dietary habits, cardiovascular risk factors and mortality in Japan. Acta Cardiol 1990; 45: 311-327.

11. Wood D, De Backer G, Faergeman O, Graham I, Mancia G, Pyörälä $\mathrm{K}$, et al. Prevention of coronary heart disease in clinical practice: Recommendations of the Second Joint Task Force of European and other societies on coronary prevention. Eur Heart J 1998; 19: 1434 1503.

12. 1996 National Heart Foundation of New Zealand Dyslipidaemia Advisory Group. Clinical guidelines for the assessment and management of dyslipidaemia: Dyslipidaemia Advisory Group on behalf of the scientific committee of the National Heart Foundation of New Zealand. NZ Med J 1996; 109: 224-231.

13. Wallis EJ, Ramsay LE, Haq IU, Ghahramani P, Jackson PR, RowlanYeo $\mathrm{K}$, et al. Coronary and cardiovascular risk estimation for primary prevention: Validation of a new Sheffield table in the 1995 Scottish health survey population. BMJ 2000; 320: 671-676.
14. Okayama A, Kadowaki T, Okamura T, Hayakawa T, Ueshima H, The NIPPON DATA80 Research Group. Age-specific effects of systolic and diastolic blood pressures on mortality due to cardiovascular diseases among Japanese men (NIPPON DATA80). J Hypertens 2006; 24: 459-462.

15. World Health Organization. International Classification of Diseases and Related Health Problems, $10^{\text {th }}$ Revision, Version for 2003. Available at: http://www3.who.int/icd/vol1htm2003/fr-icd.htm (accessed May 2, 2006).

16. Nakamura M, Sato S, Shimamoto T. Improvement in Japanese clinical laboratory measurements of total cholesterol and HDL-cholesterol by the US Cholesterol Reference Method Laboratory Network. $J$ Atheroscler Thromb 2003; 10: 145-153.

17. Bittner D, McCleary M. The cupric-phenoanthroline chelate in the determination of monosaccharides in whole blood. Am J Clin Pathol 1963; 40: 423-424.

18. Iso H, Imano H, Kitamura A, Sato S, Naito $\mathrm{Y}$, Tanigawa $\mathrm{T}$, et al. Type 2 diabetes and risk of non-embolic ischaemic stroke in Japanese men and women. Diabetologia 2004; 47: 2137-2144.

19. Kasagi F, Kodama K, Hayakawa T, Okayama A, Ueshima H, NIPPON DATA80 Research Group. Risk appraisal chart based on NIPPON DATA80: Stroke and coronary heart disease. Jpn J Cerebro-cardiovasc Dis Prev 2005; 40: 20-26 (in Japanese).

20. Japanese Society of Hypertension and Guideline Subcommittee of JSH. Guideline for the treatment of hypertension 2004. Tokyo: Life Science, 2004 (in Japanese).

21. Japan Atherosclerosis Society. Japan Atherosclerosis Society (JAS) Guideline for Diagnosis and Treatment of Atherosclerotic Cardiovascular Diseases. Tokyo: MDS, 2002 (in Japanese).

22. Noda H, Iso H, Sairenchi T, Irie F, Fukasawa N, Toriyama Y, et al. Prediction of stroke, coronary heart disease, cardiovascular disease, cancer and total death based on results of annual health checkups. Nippon Koshu Eisei Zasshi 2006; 53: 265-276 (in Japanese).

23. Mannami T, Iso H, Baba S, Sasaki S, Okada K, Konishi M, et al for the Japan Public Health Center-based Prospective Study on Cancer and Cardiovascular Disease Group. Cigarette smoking and risk of stroke and its subtypes among middle-aged Japanese men and women: The JPHC Study Cohort I. Stroke 2004; 35: 1248-1253.

24. Kubo M, Kiyohara Y, Kato I, Tanizaki Y, Arima H, Tanaka K, et al. Trends in the incidence, mortality, and survival rate of cardiovascular disease in a Japanese community: The Hisayama study. Stroke 2003; 34: 2349-2354.

25. Kodama K, Sasaki H, Shimizu Y. Trend of coronary heart disease and its relationship to risk factors in a Japanese population: A 26-year follow-up, Hiroshima/Nagasaki study. Jpn Circ J 1990; 54: 414-421.

26. Nakayama T, Date C, Yokoyama T, Yoshiike N, Yamaguchi M, Tanaka H. A 15.5-year follow-up study of stroke in a Japanese provincial city: The Shibata Study. Stroke 1997; 28: 45-52.

27. Morikawa Y, Nakagawa H, Naruse Y, Nishijo M, Miura K, Tabata $\mathrm{M}$, et al. Trends in stroke incidence and acute case fatality in a Japanese rural area: The Oyabe study. Stroke 2000; 31: 1583-1587.

28. Kitamura A, Iso H, Sankai T, Naito Y, Sato S, Kiyama M, et al. Alcohol intake and premature coronary heart disease in urban Japanese men. Am J Epidemiol 1998; 147: 59-65.

29. Shimamoto T, Komachi Y, Inada H, Doi M, Iso H, Sato S, et al. Trends for coronary heart disease and stroke and their risk factors in Japan. Circulation 1989; 79: 503-515.

30. Takeuchi H, Saitoh S, Takagi S, Ohnishi H, Ohhata J, Isobe T, et al. Metabolic syndrome and cardiac disease in Japanese men: Applicability of the concept of metabolic syndrome defined by the National Cholesterol Education Program-Adult Treatment Panel III to Japanese men: The Tanno and Sobetsu Study. Hypertens Res 2005; 28: $203-208$.

31. Asayama K, Ohkubo T, Kikuya M, Metoki H, Hoshi H, Hashimoto $\mathrm{J}$, et al. Prediction of stroke by self-measurement of blood pressure at home versus casual screening blood pressure measurement in relation to the Joint National Committee 7 classification: The Ohasama study. Stroke 2004; 35: 2356-23561.

32. Tamaki J, Ueshima H, Hayakawa T, Choudhury SR, Kodama K, Kita $Y$, et al. Effect of conventional risk factors for excess cardiovascular death in men: NIPPON DATA80. Circ J 2006; 70: 370-375.

33. Tanizaki Y, Kiyohara Y, Kato I, Iwamoto H, Nakayama K, Shinohara $\mathrm{N}$, et al. Incidence and risk factors for subtypes of cerebral infarction in a general population: The Hisayama Study. Stroke 2000; 31: $2616-2622$.

34. Ueshima H, Iida M, Shimamoto T, Konishi M, Tsujioka K, Tanigaki M, et al. Multivariate analysis of risk factors for stroke: Eight-year follow-up study of farming villages in Akita, Japan. Prev Med 1980; 9: $722-740$

35. Lin CH, Shimizu Y, Kato H, Robertson TL, Furonaka H, Kodama K, 
et al. Cerebrovascular diseases in a fixed population of Hiroshima and Nagasaki, with special reference to relationship between type and risk factors. Stroke 1984; 15: 653-660.

\section{Appendix 1}

\section{Members of NIPPON DATA80 Research Group}

Writing Group Members

H Ueshima (Department of Health Science, Shiga University of Medical Science; F Kasagi (Department of Epidemiology, Radiation Effects Research Foundation); K Kodama,(Department of Epidemiology, Radiation Effects Research Foundation); T Okamura (Department of Health Science, Shiga University of Medical Science); T Hayakawa (Department of Public Health, School of Medicine, Shimane University); A Okayama (Department of Preventive Cardiology, National Cardiovascular Center). NIPPON DATA80: National Integrated Project for Prospective Observation of Non-Communicable Disease And its Trends in the Aged

Chairman: Hirotsugu Ueshima (Department of Health Science, Shiga University of Medical Science, Otsu, Shiga)

Consultants: Osamu Iimura (Hokkaido JR Sapporo Hospital, Sapporo,

Hokkaido); Teruo Omae (Health C\&C Center Hisayama, Kasuya, Fukuoka); Kazuo Ueda (Murakami Memorial Hospital, Nakatsu, Oita);

Hiroshi Yanagawa (Saitama Prefectural University, Koshigaya, Saitama); Hiroshi Horibe (Aichi Medical University, Nagakute, Aichi).

Research Members: Akira Okayama (Department of Preventive Cardiology, National Cardiovascular Center, Suita, Osaka); Kazunori Kodama, Fumiyoshi Kasagi (Radiation Effects Research Foundation, Hiroshima,
Hiroshima); Tomonori Okamura, Yoshikuni Kita (Department of Health Science, Shiga University of Medical Science, Otsu, Shiga); Takehito Hayakawa, Shinichi Tanihara (Department of Public Health, School of Medicine, Shimane University, Izumo, Shimane); Shigeyuki Saito (Second Department of Internal Medicine School of Medicine, Sapporo Medical University, Sapporo, Hokkaido); Kiyomi Sakata (Department of Hygiene and Preventive Medicine, Iwate Medical University School of Medicine, Morioka, Iwate); Yosikazu Nakamura (Department of Health Science Division of Epidemiology and Community Health, Jichi Medical School, Minami Kawachi, Tochigi); Fumihiko Kakuno (Hikone Public Health Center, Hikone, Shiga).

Research Associate Members: Toshihiro Takeuchi, Mitsuru Hasebe, Fumitsugu Kusano, Takahisa Kawamoto and members of 300 Public Health Centers in Japan, Masumi Minowa (Faculty of Humanities, Seitoku University, Matsudo, Chiba); Katsuhiko Kawaminami (Department of Public Health Policy, National Institute of Public Health, Wako, Saitama); Sohel R. Choudhury (National Heart Foundation Hospital \& Research Institute, Dhaka, Bangladesh); Yutaka Kiyohara (Department of Medicine and Clinical Science, Graduate School of Medical Sciences, Kyushu University, Fukuoka, Fukuoka); Minoru Iida (Kansai University of Welfare Sciences, Kashihara, Kashiwara Osaka); Tsutomu Hashimoto (Kinugasa General Hospital, Yokosuka, Kanagawa); Atsushi Terao (Health Promotion Division, Department of Public Health and Welfare, Shiga Prefecture, Otsu, Shiga); Koryo Sawai (The Japanese Association for Cerebro-cardiovascular Disease Control, Tokyo); Shigeo Shibata (Clinical Nutrition, Kagawa Nutrition University, Sakado, Saitama). 